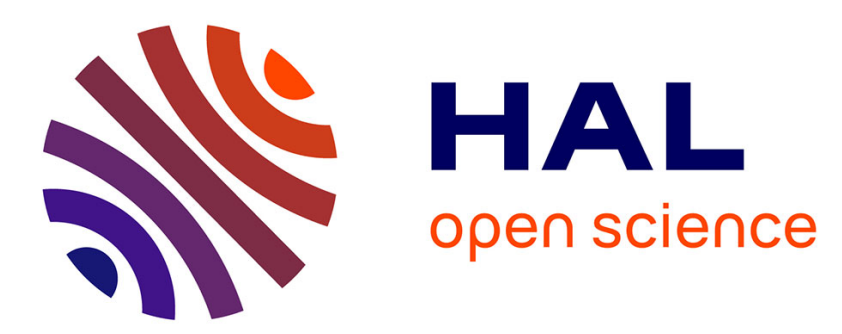

\title{
Anisotropie des propriétés magnétiques d'un atome “ habillé " par des photons de RF
}

C. Landré, C. Cohen-Tannoudji, J. Dupont-Roc, Serge Haroche

\section{To cite this version:}

C. Landré, C. Cohen-Tannoudji, J. Dupont-Roc, Serge Haroche. Anisotropie des propriétés magnétiques d'un atome " habillé " par des photons de RF. Journal de Physique, 1970, 31 (11-12), pp.971-983. 10.1051/jphys:019700031011-12097100 . jpa-00207011

\section{HAL Id: jpa-00207011 https://hal.science/jpa-00207011}

Submitted on 1 Jan 1970

HAL is a multi-disciplinary open access archive for the deposit and dissemination of scientific research documents, whether they are published or not. The documents may come from teaching and research institutions in France or abroad, or from public or private research centers.
L'archive ouverte pluridisciplinaire HAL, est destinée au dépôt et à la diffusion de documents scientifiques de niveau recherche, publiés ou non, émanant des établissements d'enseignement et de recherche français ou étrangers, des laboratoires publics ou privés. 


\title{
ANISOTROPIE DES PROPRIÉTÉS MAGNÉTIQUES D'UN ATOME «HABILLÉ » PAR DES PHOTONS DE RF (*)
}

\author{
C. LANDRÉ, C. COHEN-TANNOUDJI, J. DUPONT-ROC et Serge HAROCHE
}

Laboratoire de Spectroscopie Hertzienne de l'E. N. S., associé au C. N. R. S., Faculté des Sciences de Paris, France

(Reçu le 3 août 1970)

\begin{abstract}
Résumé. - On montre théoriquement et expérimentalement que les propriétés magnétiques d'un atome ( habillé ) par un champ de radiofréquence linéaire et non résonnant $\mathbf{H}_{1} \cos \omega t$ sont anisotropes : le facteur de Landé dépend de l'angle entre le champ statique $\mathbf{H}_{0}$ et $\mathbf{H}_{1}$; la précession de Larmor n'est plus circulaire mais elliptique. On introduit un formalisme opératoriel commode, qui permet d'écrire simplement l'équation pilote du problème et d'interpréter quantitativement les divers effets précédents.
\end{abstract}

Abstract. - It is shown theoretically and experimentally that the (c dressing ) by a non resonant linear RF field $\mathbf{H}_{1} \cos \omega \mathrm{t}$ introduces an anisotropy in the magnetic properties of an atomic system : the Landé factor depends on the angle between the static fields $\mathbf{H}_{0}$ and $\mathbf{H}_{1}$; the Larmor precession is no more circular but elliptical. A convenient operator formalism is developed from which the master equation of the problem is simply deduced, and a quantitative treatment of the previous effects obtained.

Introduction. - De nombreux travaux [1, 2, 3, 4] ont montré l'intérêt qu'il y avait à considérer, dans les expériences de résonance magnétique, le système global formé par l'atome et la radiofréquence. Les propriétés de ce système global, appelé encore atome " habillé » permettent de rendre compte très simplement des différents effets liés à l'interaction entre un système atomique et un champ de radiofréquence : dans le cas où l'on utilise les méthodes optiques de détection de la résonance magnétique, on peut par exemple considérer que c'est l'atome "habillé » qui absorbe et diffuse la lumière incidente. L'étude de son diagramme d'énergie révèle l'existence de nombreux croisements et anticroisements de niveaux qui permettent de classer de façon très synthétique les diverses résonances apparaissant en pompage optique longitudinal ou transversal $[1,2]$; d'interpréter très simplement des effets tels que l'effet Autler-Townes [5]...

Il se trouve également que lorsque le champ de RF est intense les propriétés magnétiques en champ statique faible de cet atome "habillé » sont profondément différentes de celles de l'atome «nu ». Pour un champ statique $\mathbf{H}_{0}$ perpendiculaire au champ de RF $\mathbf{H}_{1} \cos \omega \mathrm{t}$ (supposé rectiligne) on trouve pour l'atome habillé des niveaux dont la pente en fonction de $\mathbf{H}_{0}$ diffère profondément de celle de l'atome "nu » [6]. Pour certaines valeurs de $\mathbf{H}_{1}$ elle peut même s'annuler.

$\left(^{*}\right)$ Cet article expose une partie des résultats théoriques et expérimentaux contenus dans la thèse de $3^{\mathrm{e}}$ cycle de $\mathrm{C}$. Landré.
Ces effets ont été observés expérimentalement et ont conduit à de nombreuses applications $[6,7,8]$.

Le couplage entre l'atome et la RF en champ statique faible n'a pas pour seul effet de changer l'énergie des niveaux ; il modifie également les fonctions d'ondes. Nous nous proposons de montrer dans cet article comment cette modification se traduit physiquement par une anisotropie des propriétés magnétiques de l'atome " habillé ». Nous introduisons ainsi un tenseur de Landé qui permet de rendre compte du fait que la pente des niveaux dépend de l'angle entre $\mathbf{H}_{0}$ et $\mathbf{H}_{1}$; nous montrons également que la précession de Larmor de l'atome "habillé » est en général elliptique et non circulaire. Tous ces phénomènes sont liés au fait que le champ $\mathbf{H}_{1} \cos \omega \mathrm{t}$ introduit une direction privilégiée dans l'espace.

Pour mener à bien le calcul de ces effets nous introduisons une méthode opératorielle particulièrement commode qui permet de tenir compte simultanément de la modification des énergies et des fonctions d'ondes et qui conduit à des constructions géométriques très simples. Cette méthode opératorielle se révèle également très utile pour l'établissement des " équations de Bloch " de l'atome "habillé ", dont la nécessité se fait sentir dès qu'il s'agit d'interpréter des résultats expérimentaux. De telles équations contiennent en effet non seulement les termes décrivant la précession de l'atome " habillé » dans le champ $\mathbf{H}_{0}$, mais encore ceux qui rendent compte de sa préparation (par pompage optique par exemple) et de sa relaxation. 
Nous commençons au paragraphe A par quelques rappels sur le formalisme de l'atome "habillé " en champ statique faible et nous introduisons la notion d'observables de l'atome "habillé ». Puis au paragraphe B nous étudions l'effet Zeeman de l'atome " habillé ", ce qui nous conduit à l'introduction d'un facteur de Landé à caractère tensoriel. Nous voyons au paragraphe $\mathrm{C}$ les conséquences de l'anisotropie de ce facteur de Landé sur l'évolution dans un champ magnétique du moment magnétique de l'atome " habillé ». Au paragraphe $\mathrm{D}$ nous tenons compte des autres causes d'évolution de ce moment magnétique (préparation-relaxation) ce qui nous conduit à l'équation pilote de l'atome « habillé » dont la résolution est nécessaire pour le calcul des expériences décrites au paragraphe $\mathrm{E}$.

A. Observables de l'atome «habillé " en champ statique faible. - 1) RAPPELS SUR L'ATOME « HABILLÉ » EN CHAMP NUL. - Nous supposons le champ de RF $\mathbf{H}_{1} \cos \omega \mathrm{t}$ parallèle à $\mathrm{Oz}$. L'hamiltonien de l'atome « habillé » s'écrit alors (en prenant $\hbar=1$ )

$$
\mathscr{H}_{0}=\omega a^{+} a+\lambda S_{z}\left(a+a^{+}\right)
$$

où $a^{+}$et $a$ sont les opérateurs de création et d'annihilation d'un photon de radiofréquence, $S_{z}$ la composante du spin atomique le long de $\mathrm{Oz}, \lambda$ une constante de couplage. $\lambda$ intervient dans la formule qui relie le nombre moyen $\langle n\rangle$ de photons à l'amplitude moyenne du champ de radiofréquence $\left\langle H_{1}\right\rangle$

$$
\gamma\left\langle H_{1}\right\rangle=\left\langle\omega_{1}\right\rangle=2 \lambda \sqrt{\langle n\rangle}
$$

(voir par exemple référence [9]), $\gamma$ est le rapport gyromagnétique du niveau atomique.

L'hamiltonien $(A, 1)$ a été étudié en détail par ailleurs $[2,9]$. Ses états propres et valeurs propres sont donnés par l'équation

$$
\mathscr{H}_{0}|\overline{m, n}>=n \omega| \overline{m, n}>.
$$

Les états $|\overline{m, n}\rangle$ sont produits tensoriels d'un état atomique $\mid m>$ (état propre de $S_{z}$ de valeur propre $m$ ) par un état de radiofréquence $\left|\bar{n}_{m}\right\rangle$ obtenu par action d'un « opérateur de déplacement» [10] sur un état $|n\rangle$ à $n$ photons (état propre de $a^{+} a$ de valeur propre $n$ )

$$
\begin{array}{rlrl}
\mid \overline{m, n}> & =|m>| \bar{n}_{m}> & (\mathrm{A}, 4 a) \\
S_{z} \mid m> & =m \mid m> & (\mathrm{A}, 4 b) \\
\mid \bar{n}_{m}> & =\exp \left[-\lambda \frac{m}{\omega}\left(a^{+}-a\right)\right] \mid n>\quad(\mathrm{A}, 4 c) \\
a^{+} a \mid n> & =n \mid n>
\end{array}
$$

On constate ainsi que les états propres de $\mathcal{H}_{0}$ se groupent en multiplicités $\varepsilon_{n}$ distantes de $\omega,(2 \mathrm{~S}+1)$ fois dégénérées. Le diagramme d'énergie de l'atome « habillé » rappelle par certains aspects celui d'un atome «nu » qui aurait un grand nombre de niveaux hyperfins $\left(^{1}\right)$.

Rappelons enfin la formule suivante établie dans la référence [9] et valable pour $n$ et $n^{\prime}$ suffisamment grands

$$
<\bar{n}_{m} \mid \bar{n}_{m^{\prime}}^{\prime}>=J_{n-n^{\prime}}\left[\left(m-m^{\prime}\right) \frac{\omega_{1}}{\omega}\right]
$$

où $J_{n-n^{\prime}}$ est la fonction de Bessel d'ordre $n-n^{\prime}$.

2) Définition DeS Opérateurs ${ }^{n n^{\prime}} \mathrm{S}$. - Soit $P_{n}$ le projecteur sur la multiplicité $\delta_{n}$ d'énergie $n \omega$

$$
P_{n}=\sum_{m=-S}^{+S}|\overline{m, n}><\overline{m, n}|
$$

Les $|\overline{m, n}\rangle$ formant une base dans l'espace des états, on a évidemment

$$
\left\{\begin{array}{l}
P_{n} P_{n^{\prime}}=\delta_{n n^{\prime}} P_{n} \\
\sum_{n} P_{n}=1
\end{array}\right.
$$

En utilisant $(\mathrm{A}, 7 \mathrm{~b})$ on peut récrire l'opérateur moment cinétique atomique $\mathbf{S}$ sous la forme :

$$
\mathbf{S}=\sum_{n n^{\prime}} P_{n} \mathbf{S} P_{n^{\prime}}=\sum_{n n^{\prime}}{ }^{n n^{\prime}} \mathbf{S}
$$

en posant

$$
{ }^{n} \mathbf{S}=P_{n} \mathbf{S} P_{n^{\prime}} .
$$

Nous verrons plus loin le rôle important joué par les opérateurs ${ }^{n n^{\prime}} \mathbf{S}$, soit pour le calcul de l'effet d'un champ statique (Tenseur de Landé de l'atome « habillé »; dans ce cas ce sont les opérateurs "diagonaux $»{ }^{n n} \mathrm{~S}$ qui interviennent) ; soit pour le calcul des signaux de détection.

Notons l'analogie entre les opérateurs ${ }^{n n} \mathbf{S}$ que nous venons de définir et des opérateurs utilisés couramment en physique atomique [11] lorsqu'un atome possède plusieurs niveaux hyperfins : ${ }^{\mathrm{FF}} \mathbf{S}$ projection du spin électronique $\mathbf{S}$ dans le niveau hyperfin $\mathbf{F}$, ${ }^{\text {FF' }} \mathbf{S}$ projection de $S$ entre sous-niveaux hyperfins distincts $\mathrm{F}$ et $\mathrm{F}^{\prime}$.

Remarquons enfin qu'en champ nul les opérateurs ${ }^{n n} \mathbf{S}$ ont une fréquence d'évolution nulle : ce sont des observables statiques. Par contre les ${ }^{n n^{\prime}} \mathbf{S}$ évoluent aux fréquences $\left(n-n^{\prime}\right) \omega$ : En champ faible, les ${ }^{n n} S$ et ${ }^{n}{ }^{\prime} \mathbf{S}$ représentent respectivement les composantes basse et haute fréquence du moment cinétique de l'atome « habillé ».

B. Tenseur de Landé de l'atome « habillé ». 1) Moment Cinétique « FICTIF » $\mathbf{S}$ DE L'Atome

(1) Cette analogie nous sera utile pour la suite mais ne doit cependant pas être poussée trop loin : en effet les différents niveaux hyperfins d'un atome sont en nombre fini, ne sont pas équidistants et ne contiennent pas tous le même nombre de sous-niveaux. 
« HABILLÉ ». - En présence d'un champ magnétique $\mathbf{H}_{0}$, le hamiltonien Zeeman du niveau atomique est

$$
V=-g \mu_{\mathbf{B}} \mathbf{S} \cdot \mathbf{H}_{0}
$$

( $g$ est le facteur de Landé du niveau, $\mu_{\mathrm{B}}$ le magnéton de Bohr, on a $g \mu_{\mathrm{B}}=\gamma$ ). Nous supposons le champ magnétique suffisamment faible pour que $\mathrm{V}$ puisse être considérée comme une perturbation du hamiltonien $\mathscr{H}_{0}\left(\omega_{0}=-g \mu_{\mathrm{B}} H_{0} \ll \omega\right)$ : la levée de dégénérescence sous l'effet du champ magnétique des différentes multiplicités de l'atome « habillé » $\varepsilon_{n}$ s'obtiendra donc au premier ordre en diagonalisant la projection ${ }^{n n} V$ de $V$ dans $\varepsilon_{n}$ :

$$
{ }^{n n} V=-g \mu_{\mathrm{B}} \mathbf{H}_{0} P_{n} \mathbf{S} P_{n}
$$

L'étude des propriétés magnétiques de l'atome " habillé » nécessite donc la connaissance des éléments de matrice de l'opérateur ${ }^{n n} \mathbf{S}$ entre les états propres de l'atome « habillé »:

$$
\begin{aligned}
& <\overline{n m}\left|{ }^{n n} S_{z}\right| \overline{n m^{\prime}}>= \\
& \quad=<m\left|S_{z}\right| m^{\prime}><\bar{n}_{m} \mid \bar{n}_{m^{\prime}}> \\
& <\overline{n m}\left|{ }^{n n} S_{y}\right| \overline{n m^{\prime}}>= \\
& \quad=<m\left|S_{y}\right| m^{\prime}><\bar{n}_{m} \mid \bar{n}_{m^{\prime}}>
\end{aligned}
$$

L'élément de matrice de $S_{z}$ au second membre de (B, $3 a$ ) impose la condition $m-m^{\prime}=0$; on a alors $\left\langle\bar{n}_{m} \mid \bar{n}_{m^{\prime}}\right\rangle=1$ dans cette relation. L'élément de matrice de $S_{x}$ au second membre de $(\mathrm{B}, 3 b)$ impose par contre la condition $m-m^{\prime}= \pm 1$; on a alors

$$
<\bar{n}_{m} \mid \bar{n}_{m^{\prime}}>=J_{0}\left(\frac{\omega_{1}}{\omega}\right)
$$

au second membre de (B, $3 b)$. On obtient donc finalement

$$
\begin{aligned}
& <\overline{n m}\left|{ }^{n n} S_{z}\right| \overline{n m^{\prime}}>=<m\left|S_{z}\right| m^{\prime}>\quad \\
& <\overline{n m}\left|{ }^{n n} S_{x}\right| \overline{n m^{\prime}}>=<m\left|S_{x}\right| m^{\prime}>J_{0}\left(\frac{\omega_{1}}{\omega}\right) .
\end{aligned}
$$

Les éléments de matrice de ${ }^{n n} \mathbf{S}$ entre états propres de l'atome " habillé » sont donc différents des éléments de matrice correspondants du moment cinétique d'un atome libre. Il en résulte que les composantes de ${ }^{n n} \mathbf{S}$ n'obéissent pas aux relations de commutation classiques du moment cinétique. Nous verrons que ce résultat est à l'origine des propriétés particulières d'anisotropie du moment magnétique de l'atome " habillé ». La complication qui apparaît ainsi dans les relations de commutation des composantes de $\mathbf{S}$ rend cet opérateur d'un emploi peu commode et conduit à introduire un nouvel opérateur vectoriel ${ }^{n n} \boldsymbol{S}$ agissant à l'intérieur de $\varepsilon_{n}$, qui possède entre les états propres de l'atome "habillé » les mêmes éléments de matrice que le moment cinétique $\mathbf{S}$ entre les états propres correspondants de l'atome libre :

$$
<\left.\overline{n m}\right|^{n n} \mathbf{S}\left|\overline{n m^{\prime}}\right\rangle=\left\langle m|\mathbf{S}| m^{\prime}\right\rangle .
$$

Par définition les composantes de ce nouvel opérateur ${ }^{n n} \boldsymbol{S}$ obéissent aux relations de commutation classiques du moment cinétique :

$$
\left[{ }^{n n} S_{x},{ }^{n n} S_{y}\right]=i^{n n} S_{z}
$$

et sont reliées aux composantes du vrai moment cinétique $\mathbf{S}$ par les relations :

$$
\left\{\begin{array}{l}
{ }^{n n} S_{z}={ }^{n} S_{z} \\
{ }^{n n} S_{y}={ }^{n n} S_{y} J_{0}\left(\frac{\omega_{1}}{\omega}\right) .
\end{array}\right.
$$

Pour le distinguer de ${ }^{n n} \mathbf{S}$, nous appellerons ${ }^{n n} \boldsymbol{S}$ le moment cinétique fictif de l'atome " habillé». Nous allons voir que la simplicité des relations de commutation $(\mathrm{B}, 6)$ rend cet opérateur d'un emploi très commode pour le calcul de l'évolution du système.

2) ANisotropie du FACTEUR de LANDÉ DE L'ATOME « HABILLÉ ». - Le hamiltonien Zeeman ${ }^{n n} V$ s'écrit en fonction des composantes de $\mathbf{H}_{0}$ et de ${ }^{n n} \mathbf{S}$ :

$$
\begin{aligned}
{ }^{n n} V=-g \mu_{\mathrm{B}}\left[H_{0 z}{ }^{n n} S_{z}\right. & +H_{0 x}{ }^{n n} S_{x} J_{0}\left(\frac{\omega_{1}}{\omega}\right)+ \\
& \left.+H_{0 y}{ }^{n n} S_{y} J_{0}\left(\frac{\omega_{1}}{\omega}\right)\right]
\end{aligned}
$$

Les niveaux d'énergie varient linéairement en fonction de $H_{0}$, ce qui permet de définir un facteur de Landé de l'atome " habillé ». Il apparaît cependant que ce facteur de Landé est anisotrope : Les valeurs propres des composantes de ${ }^{n n} \boldsymbol{S}$ étant les mêmes que celles d'un moment cinétique ordinaire, on voit aisément que ce facteur de Landé est égal à celui de l'atome libre, $g$, si $\mathbf{H}_{0}$ est parallèle à $O z$, à $g J_{0}\left(\frac{\omega_{1}}{\omega}\right)$ si $\mathbf{H}_{0}$ est perpendiculaire à $\mathrm{Oz}$. Il s'annule alors dans ce dernier cas pour toutes les valeurs de $\omega_{1}$ correspondant à un zéro de $J_{0}$ [6]. Ainsi le champ de radiofréquence, en introduisant dans le système une direction privilégiée a rendu anisotrope les propriétés magnétiques de l'atome " habillé ». On peut rendre compte de cette anisotropie en définissant un tenseur de Landé $\bar{g}$ de l'atome " habillé » de composantes $\bar{g}_{i j}(i, j=x$, $y, z)$. Rapporté aux axes Oxyz, ce tenseur est diagonal et possède la symétrie de révolution autour de $\mathrm{Oz}$ :

$$
\bar{g}=g\left[\begin{array}{ccc}
J_{0}\left(\frac{\omega_{1}}{\omega}\right) & 0 & 0 \\
0 & J_{0}\left(\frac{\omega_{1}}{\omega}\right) & 0 \\
0 & 0 & 1
\end{array}\right]
$$


L'énergie magnétique s'écrit alors de façon générale $\left({ }^{2}\right)$ :

$$
{ }^{n n} V=-\mu_{\mathrm{B}} \bar{g}_{i j} H_{0 i}{ }^{n} \mathrm{~S}_{j} .
$$

Nous allons voir l'intérêt qu'il y a, pour diagonaliser ${ }^{n n} V$, à introduire la notion de champ magnétique fictif.

3) Problème équivalent : atome libre dans un CHAMP MAGNÉTIQUE FICTIF. - Le hamiltonien Zeeman $(B, 10)$ est formellement identique à celui d'un atome libre plongé dans un champ magnétique fictif $\mathbf{H}_{0}^{\prime}$ de composantes

$$
\left\{\begin{array}{l}
H_{0 z}^{\prime}=H_{0 z} \\
H_{0_{y}^{x}}^{\prime}=H_{0 x} J_{0}\left(\frac{\omega_{1}}{\omega}\right)
\end{array}\right.
$$

soit encore de façon générale

$$
H_{0 i}^{\prime}=\bar{g}_{i j} H_{0 j} \text {. }
$$

On a alors

$$
{ }^{n n} V=-g \mu_{\mathrm{B}} \mathbf{H}_{0}^{\prime n} \mathbf{S} .
$$

L'introduction de ce champ fictif permet de remplacer l'étude d'un système à facteur de Landé anisotrope par celle d'un atome libre de facteur de Landé isotrope, mais plongé dans un champ magnétique se déduisant du champ appliqué par une affinité orthogonale d'axe $\mathrm{Oz}$ et de rapport $J_{0}\left(\omega_{1} / \omega\right)$.

Les niveaux d'énergie dans un champ magnétique quelconque se calculent alors immédiatement: la multiplicité $\delta_{n}$ se scinde en $(2 \mathrm{~S}+1)$ sous-niveaux équidistants séparés de

$$
\begin{aligned}
\Delta E= & g \mu_{\mathrm{B}}\left|H_{0}^{\prime}\right|= \\
& =g \mu_{\mathrm{B}} \sqrt{H_{0 z}^{2}+J_{0}^{2}\left(\frac{\omega_{1}}{\omega}\right)\left(H_{0 x}^{2}+H_{0 y}^{2}\right)} \\
& =g \mu_{\mathrm{B}} \sqrt{H_{0 / /}^{2}+J_{0}^{2}\left(\frac{\omega_{1}}{\omega}\right) H_{0 \perp}^{2}}
\end{aligned}
$$

en appelant respectivement $\mathbf{H}_{0 / /}$ et $\mathbf{H}_{0 \perp}$ les composantes de $\mathbf{H}_{0}$ parallèles et perpendiculaires à la direction $\mathrm{Oz}$ du champ « habillant ».

4) RePRÉSENTATIONS GÉOMÉTRIQUES DU TENSEUR DE LANDÉ. - Nous pouvons maintenant calculer le facteur de Landé pour un champ $\mathbf{H}_{0}$ faisant un angle quelconque $\theta$ avec $\mathrm{Oz}$. On a alors

Soit

$$
H_{0 / /}=H_{0} \cos \theta \text { et } H_{0 \perp}=H_{0} \sin \theta .
$$

$$
\Delta E=g \mu_{\mathrm{B}} H_{0} \sqrt{\cos ^{2} \theta+\sin ^{2} \theta J_{0}^{2}\left(\frac{\omega_{1}}{\omega}\right)} .
$$

(2) On applique la règle de sommation d'Einstein sur les indices répétés.
Le facteur de Landé dans la direction $\theta$ est donc

$$
\bar{g}_{\theta}=g \sqrt{\cos ^{2} \theta+J_{0}^{2}\left(\frac{\omega_{1}}{\omega}\right) \sin ^{2} \theta} .
$$

Si l'on reporte dans chaque direction $(\theta, \varphi)$ une longueur proportionnelle au facteur de Landé dans cette direction, on obtient une surface de révolution autour de $\mathrm{Oz}$, dont la courbe méridienne est donnée en coordonnées polaires par l'équation $(B, 14)$ (voir Fig. 1).

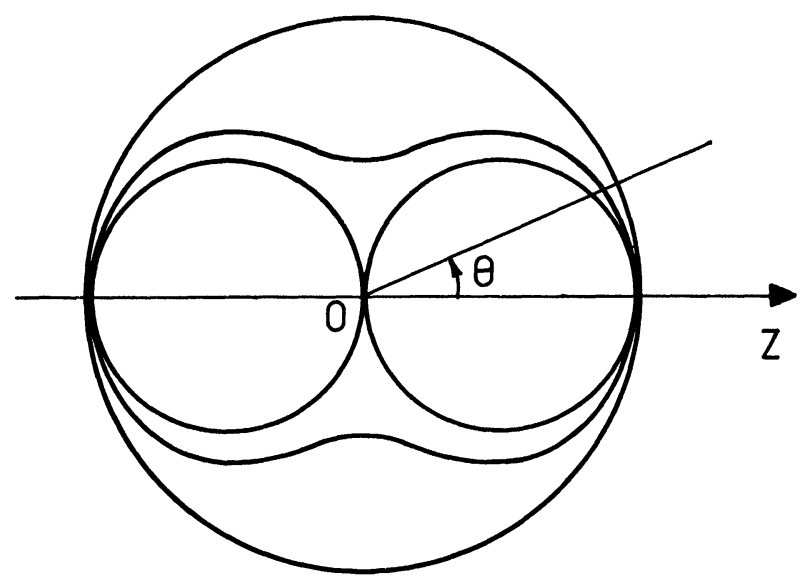

Fig. 1. - Section par un plan méridien de la surface des facteurs de Landé de l'atome "habillé » pour trois valeurs de $\omega_{1} / \omega$

$$
\left(\frac{\omega_{1}}{\omega}=0 ; \frac{\omega_{1}}{\omega}=1,6 ; \frac{\omega_{1}}{\omega}=2,4\right) .
$$

Si le champ de radiofréquence est nul, $J_{0}\left(\omega_{1} / \omega\right)=1$ et la surface est évidemment une sphère décrivant le facteur de Landé isotrope de l'atome libre; pour les valeurs de $\omega_{1} / \omega$ annulant $J_{0}$, on a $\bar{g}_{\theta}=g \cos \theta$ et la surface est constituée de deux sphères tangentes au plan $\mathrm{xOy} ;$ le facteur de Landé est alors égal à celui de l'atome «nu » le long de $\mathrm{Oz}$ et nul pour un champ magnétique perpendiculaire à $\mathrm{Oz}$.

$\mathrm{Si}$ au lieu de reporter dans chaque direction $\bar{g}_{\theta}$, on reporte $\rho_{\theta}=1 / \bar{g}_{9}$ on obtient une courbe méridienne d'équation polaire

$$
\rho_{\theta}=\frac{1}{g} \frac{1}{\sqrt{\cos ^{2} \theta+J_{0}^{2}\left(\frac{\omega_{1}}{\omega}\right) \sin ^{2} \theta}}
$$

qui n'est autre dans le plan $\mathrm{xOz}$ que l'ellipse

$$
z^{2}+J_{0}^{2}\left(\frac{\omega_{1}}{\omega}\right) x^{2}=1 / g^{2} .
$$

On définit ainsi un ellipsoïde de révolution aplati, de révolution autour de Oz. On notera l'analogie de ces représentations géométriques avec celles des surfaces ou des ellipsoïdes des indices, ou d'inertie, employés communément pour représenter géométriquement l'indice de réfraction tensoriel, ou le tenseur d'inertie, d'un système physique anisotrope. 
C. Précession de Larmor de l'atome « habillé ». Après avoir étudié les niveaux d'énergie de l'atome « habillé », il nous faut maintenant préciser l'évolution de ses observables dans un champ magnétique.

1) Evolution DU MOMENT CINÉTIQUe FICTIF ${ }^{n n} \boldsymbol{S}$ DE L'ATOME « HABILLÉ ». - Nous allons établir les équations d'évolution de ${ }^{n n} \boldsymbol{S}$. Celles du moment cinétique ${ }^{n n} \mathbf{S}$ s'en déduiront par application des relations $(B, 7)$.

L'équation de Heisenberg s'écrit pour ${ }^{n n} \boldsymbol{S}$

$$
\begin{aligned}
i \frac{\mathrm{d}}{\mathrm{d} t}{ }^{n n} \mathrm{~S}_{i} & =\left[{ }^{n n} \mathrm{~S}_{i}, \mathcal{H}\right] \\
& =\left[{ }^{n n} \mathrm{~S}_{i}, V\right]
\end{aligned}
$$

(le commutateur de ${ }^{n n} \boldsymbol{S}=P_{n} \boldsymbol{S} P_{n}$ avec

$$
\mathcal{H}_{0}=\omega a^{+} a+\lambda S_{z}\left(a+a^{+}\right)
$$

est en effet nul puisque $P_{n}$ est un projecteur sur une multiplicité dégénérée d'états propres de $\mathscr{H}_{0}$ ).

Introduisant deux fois la relation de fermeture sur les projecteurs $P_{n}$, nous pouvons développer $V$ sous la forme

$$
V=\sum_{n, n^{\prime}} P_{n} V P_{n^{\prime}}
$$

Le commutateur de $(\mathrm{C}, 1)$ fait alors intervenir des opérateurs de la forme ${ }^{n n} S_{i} P_{n} V P_{n^{\prime}}$. De tels opérateurs, agissant entre les multiplicités $n$ et $n^{\prime}$ évoluent, à l'ordre zéro en $V$, à la fréquence $\left(n-n^{\prime}\right) \omega$. Si $n^{\prime} \neq n$, l'effet de ces termes sur l'évolution de ${ }^{n n} S_{i}$, statique à l'ordre zéro en $V$, est faible (approximation séculaire). Nous négligerons donc dans le développement de $V$ les projections $P_{n} V P_{n^{\prime}}\left(n^{\prime} \neq n\right)$ qui conduisent à des termes non séculaires et nous écrirons :

$$
i \frac{\mathrm{d}}{\mathrm{d} t}{ }^{n n} \mathrm{~S}_{i}=\left[{ }^{n n} \mathrm{~S}_{i},{ }^{n n} V\right]
$$

Soit encore en utilisant l'expression $(B, 12)$ de ${ }^{n n} V$ en fonction du champ fictif $\mathbf{H}_{0}^{\prime}$ :

$$
i \frac{\mathrm{d}}{\mathrm{d} t}{ }^{n} S_{i}=\left[{ }^{n n} S_{i},-g \mu_{\mathrm{B}} \mathbf{H}_{0}^{\prime}{ }^{n n} \mathrm{~S}\right] .
$$

Par définition, les relations de commutation des ${ }^{n n} S_{i}$ sont identiques à celles des composantes du moment cinétique de l'atome libre. Nous voyons alors clairement l'intérêt des notions de moment cinétique et de champ fictif précédemment introduites : au lieu d'étudier l'évolution compliquée dans le champ $\mathbf{H}_{0}$ d'un système à facteur de Landé anisotrope, nous sommes ramenés à l'étude classique de l'évolution du moment cinétique d'un atome libre dans le champ $\mathbf{H}_{0}^{\prime}$. Ainsi le mouvement du vrai moment cinétique ${ }^{n n} \mathbf{S}$ se détermine en trois étapes:

a) on effectue sur $\mathbf{H}_{0}$ l'affinité $(\mathrm{B}, 11)$ pour obtenir $\mathbf{H}_{0}^{\prime}$;

b) on calcule à l'aide de $(\mathrm{C}, 4)$ l'évolution du moment fictif ${ }^{n n} \boldsymbol{S}$ dans $\mathbf{H}_{0}^{\prime}$; c) on revient à l'aide de l'affinité $(B, 7)$ à l'observable physique ${ }^{n n} \mathbf{S}$.

2) PréCession de LARMOR DE L'ATOME « HABILLÉ ». - L'équation $(\mathrm{C}, 4)$ conduit à l'équation vectorielle pour les valeurs moyennes

$$
\frac{\mathrm{d}}{\mathrm{d} t}<{ }^{n n} \mathrm{~S}>=g \mu_{\mathrm{B}}<{ }^{n n} \mathrm{~S}>\wedge \mathbf{H}_{0}^{\prime}
$$

qui décrit la précession de Larmor de $\left\langle{ }^{n n} \boldsymbol{S}>\right.$ sur un cône de révolution autour de $\mathbf{H}_{0}^{\prime}$ à la fréquence $g \mu_{\mathrm{B}} H_{0}^{\prime}$. L'évolution de $\left\langle{ }^{n} \mathrm{~S}\right\rangle$ s'obtient en effectuant l'affinité $(\mathrm{B}, 7)$ :

a. Fréquence de Larmor. - La fréquence de précession de $\left\langle{ }^{n n} \mathbf{S}\right\rangle$ est évidemment la même que celle de $\left\langle{ }^{n n} \mathbf{S}\right\rangle$. Cette fréquence n'est plus celle de l'atome libre, $\omega_{0}=-g \mu_{\mathrm{B}} H_{0}$ mais devient

$$
\bar{\omega}_{0}=-g \mu_{\mathbf{B}} H_{0}^{\prime}=-\bar{g}_{0} \mu_{\mathbf{B}} H_{0}
$$

et correspond évidemment à la séparation en énergie des sous-niveaux Zeeman calculés au paragraphe précédent. De la mesure de la fréquence de précession pour diverses orientations de $\mathbf{H}_{0}$, on peut, à l'aide de $(C, 6)$ déduire le facteur de Landé anisotrope $\bar{g}_{\theta}$ de l'atome "habillé » et déterminer expérimentalement la surface des facteurs de Landé (voir $\S \mathrm{E}, 2$ ).

b) Forme de la précession. - La surface engendrée par le vrai moment cinétique $\left\langle{ }^{n n} \mathbf{S}\right\rangle$ se déduit par l'affinité $(\mathrm{B}, 7)$ du cône de révolution décrit par $\left\langle{ }^{n n} \mathbf{S}\right\rangle$ autour de $\mathbf{H}_{0}^{\prime}$ : appelons $\mathbf{H}_{0}^{\prime \prime}$ le transformé de $\mathbf{H}_{0}^{\prime}$ dans la même affinité

$$
\left\{\begin{array}{l}
H_{0 z}^{\prime \prime}=H_{0 z}^{\prime}=H_{0 z} \\
H_{0_{y}^{x}}^{\prime \prime}=H_{0_{y}^{x}}^{\prime} J_{0}\left(\frac{\omega_{1}}{\omega}\right)=H_{0_{y}^{x}} J_{0}^{2}\left(\frac{\omega_{1}}{\omega}\right)
\end{array}\right.
$$

$\left\langle{ }^{n n} \mathbf{S}\right\rangle$ engendre un cône qui n'est plus de révolution et son extrémité décrit une ellipse dont le centre est sur $H_{0}^{\prime \prime}$ et qui est transformée dans la même affinité $\mathrm{du}$ cercle décrit par l'extrémité de $\left\langle{ }^{n n} \boldsymbol{S}\right\rangle$ (voir Fig. 2a). D'autre part, le module du moment cinétique $\left|\left\langle{ }^{n n} \mathbf{S}\right\rangle\right|$ varie au cours de la précession : ainsi le couplage avec le champ de radiofréquence a non seulement diminué la fréquence de la précession de Larmor, mais a considérablement modifié le mouvement de précession lui-même : nous retiendrons que le moment cinétique "précesse autour » de la direction de $\mathbf{H}_{0}^{\prime \prime}$

$$
H_{0 i}^{\prime \prime}=(\bar{g})_{i j}^{2} H_{0 j}
$$

en général différente de celle du champ magnétique appliqué, que le module du moment cinétique n'est plus une constante du mouvement et que son extrémité évolue sur une ellipse et non plus sur un cercle comme pour l'atome libre. Les seuls cas particuliers où la précession s'effectue autour du champ appliqué 

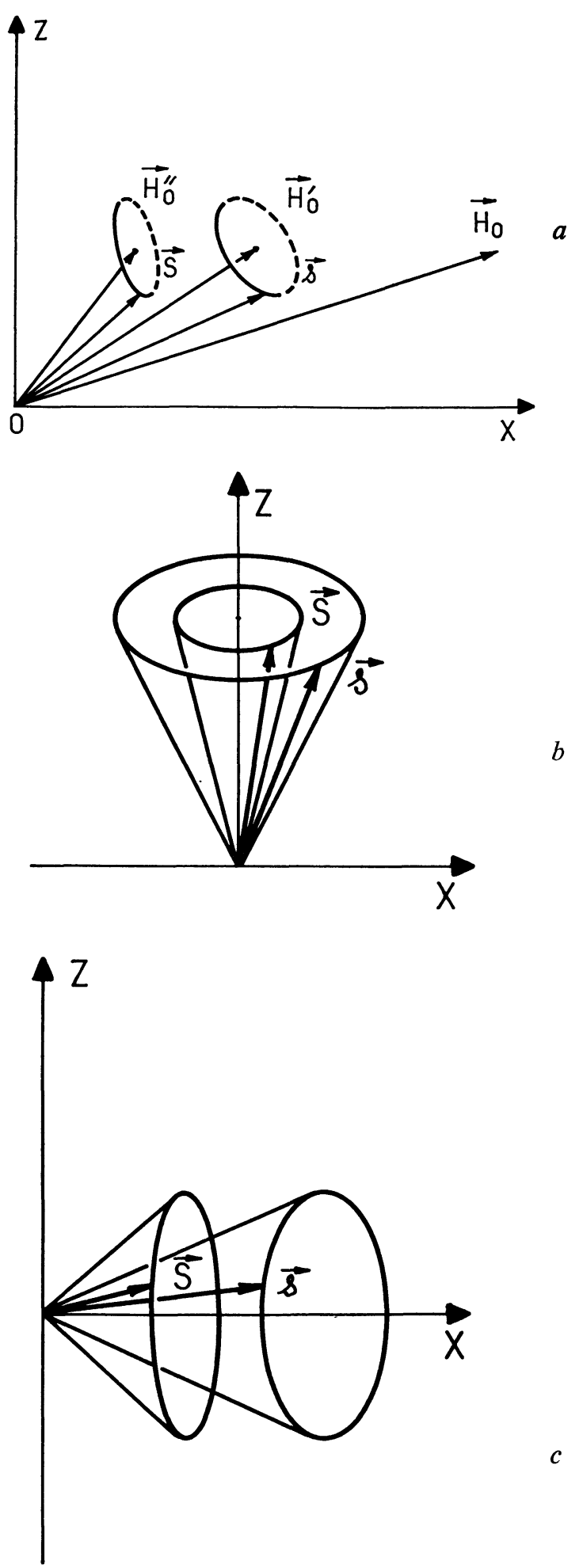

Fig. 2. - Précession du moment cinétique fictif $n n s$ et du vrai moment cinétique ${ }^{n n} \mathbf{S}$ de l'atome habillé dans un champ Hë.

a) Cas général : $\mathbf{H}_{0}$ de direction quelconque.

b) $H_{0}$ parallèle à la direction $\mathrm{Oz}$ du champ « habillant».

c) $H_{0}$ perpendiculaire à la direction du champ « habillant ».

correspondent au cas où celui-ci est orienté le long d'une des directions principales du tenseur de Landé, c'est-à-dire parallèlement ou perpendiculairement à la direction $\mathrm{Oz}$ du champ de radiofréquence :
Si $\mathbf{H}_{0}$ est parallèle à $\mathrm{Oz}$, on a

$$
\mathbf{H}_{0}^{\prime \prime}=\mathbf{H}_{0}^{\prime}=\mathbf{H}_{0} \text {. }
$$

$<{ }^{n n} \mathrm{~S}>$ précesse sur un cône de révolution d'axe $\mathrm{Oz}$ à la fréquence $\omega_{0}=-g \mu_{B} H_{0} \cdot\left\langle{ }^{n n} \mathbf{S}>\right.$ précesse alors à la même fréquence sur un cône de révolution de même axe et d'ouverture $J_{0}\left(\omega_{1} / \omega\right)$ fois plus petite. L'extrémité de $\left\langle{ }^{n n} \mathbf{S}\right\rangle$ décrit dans ce cas un cercle autour de $\mathbf{H}_{0}$ (voir Fig. $2 b$ ).

$\mathrm{Si} \mathbf{H}_{0}$ est perpendiculaire à $\mathrm{Oz}$, par exemple le long de $\mathrm{Ox}$, on a

$$
\mathbf{H}_{0}^{\prime \prime}=J_{0}\left(\frac{\omega_{1}}{\omega}\right) \mathbf{H}_{0}^{\prime}=J_{0}^{2}\left(\frac{\omega_{1}}{\omega}\right) \mathbf{H}_{0} .
$$

$<{ }^{n n} \mathrm{~S}>$ précesse sur un cône de révolution d'axe $\mathbf{O x}$ à la fréquence $\bar{\omega}_{0}=-g \mu_{\mathrm{B}} J_{0}\left(\omega_{1} / \omega\right) H_{0}$. L'extrémité de $\left\langle{ }^{n n} \mathrm{~S}\right\rangle$ décrit alors à la même fréquence une ellipse centrée sur $\mathrm{Ox}$, de grand axe parallèle à $\mathrm{Oz}$, de petit axe parallèle à $O y$, le rapport des axes valant $J_{0}\left(\omega_{1} / \omega\right)$ (voir Fig. 2c). Nous montrons au $\S \mathrm{E}, 3$ ) comment on peut étudier expérimentalement ce mouvement.

D. Equation pilote de l'atome « habillé ». 1) Position du PROBlÈME. - L'équation $(\mathrm{C}, 5)$ donne la vitesse de variation de $\left\langle{ }^{n n} \boldsymbol{S}\right\rangle$ due à la précession de Larmor. En utilisant les notations de la référence [12], nous noterons $\mathrm{d}^{(3)} / \mathrm{d} t<{ }^{n n} \mathbf{S}>$ cette vitesse de variation.

Il nous faut encore pour calculer $\left\langle{ }^{n n} \mathbf{S}\right\rangle$ à tout instant $t$ déterminer les vitesses de variations de $\left\langle{ }^{n n} \boldsymbol{S}\right\rangle$ dues aux autres processus physiques qui entrent en jeu : pompage ou encore préparation du système, relaxation. Conformément aux notations de la référence [12] nous noterons $\mathrm{d}^{(1)} / \mathrm{d} t\left\langle{ }^{n n} \mathbf{S}\right\rangle$ et $\mathrm{d}^{(2)} / \mathrm{d} t<{ }^{n n} \boldsymbol{S}>$ ces vitesses de variation.

L'évolution globale de $\left\langle{ }^{n n} \boldsymbol{S}>\right.$ s'obtient en ajoutant les 3 vitesses de variation précédentes. L'équation pilote ainsi obtenue peut encore être considérée comme l'équation de Bloch de l'atome « habillé ".

Enfin l'interprétation des résultats expérimentaux nécessite de relier les signaux de détection aux grandeurs $\left\langle{ }^{n n} \boldsymbol{S}\right\rangle$ calculées à partir de l'équation de Bloch.

Remarque. - Nous! nous limitons ici pour simplifier, à l'étude des observables basse fréquence $\left\langle{ }^{n n} \boldsymbol{S}\right\rangle$. La généralisation aux grandeurs haute fréquence du type $\left\langle{ }^{n n} \boldsymbol{S}\right\rangle$ est faite dans la référence [13]. On y étudie également l'évolution d'opérateurs tensoriels irréductibles $<{ }^{n n^{\prime}} T_{q}^{(k)}>$.

2) Préparation ou POMPage dU Système. a) Cas d'un atome libre. - Considérons pour fixer les idées le cas où $\mathbf{S}$ est le moment cinétique de l'état excité d'un atome libre, atteint par excitation optique à partir d'un état fondamental. Le terme qui décrit la préparation de $\langle\mathbf{S}\rangle$ ou encore le terme source de l'équation pilote s'écrit 


$$
\frac{\mathrm{d}^{(1)}}{\mathrm{d} t}<\mathrm{S}>=\frac{1}{T_{\mathrm{p}}}{ }^{\mathrm{ex}} \mathrm{S} .
$$

$T_{\mathrm{p}}$ est un paramètre appelé temps de pompage d'autant plus court que l'intensité lumineuse est plus grande : ${ }^{\text {ex }} \mathrm{S}$ un vecteur dont les composantes ${ }^{\mathrm{ex}} S_{x}$, ${ }^{\mathrm{ex}} S_{y}$, ${ }^{\mathrm{ex}} S_{z}$ dépendent de la direction et de la polarisation du faisceau lumineux. Si $\sigma_{0}$ est la matrice densité d'un atome qui vient juste d'être excité, on a

$$
{ }^{\text {ex }} \mathbf{S}=\operatorname{Tr} \sigma_{0} \mathbf{S} .
$$

b) Résultat pour l'atome habillé. - Il nous faut maintenant trouver l'équivalent de $(D, 1)$ lorsque l'atome interagit dans l'état excité avec un champ de $\mathrm{RF}$ dirigé suivant $\mathrm{Oz}$.

Le processus d'excitation optique dure en général un temps très court, de l'ordre de $1 / \Delta$ où $\Delta$ est la largeur spectrale de la raie excitatrice. $\Delta$ est la plupart du temps très grand devant $\omega, \omega_{1}=-\gamma H_{1}$ de sorte que pendant la durée du processus d'absorption on peut négliger le couplage entre l'atome et la radiofréquence. Immédiatement après l'absorption du photon optique que l'on peut ainsi considérer comme instantanée la matrice densité du système global, « atome + RF » s'écrit donc

$$
{ }^{\mathrm{ex}} \sigma=\sigma_{0} \otimes \sigma_{\mathrm{RF}}
$$

où $\sigma_{0}$ a été défini plus haut et où $\sigma_{\mathrm{RF}}$ est la matrice densité associée au champ de RF. (Les deux systèmes sont brusquement mis en présence et n'ont pas encore commencé à interagir.) Nous prenons pour $\sigma_{\mathrm{RF}}$ une matrice densité correspondant à un état cohérent de Glauber [10]. Rappelons que si $\langle n\rangle$ est le nombre moyen de photons dans un tel état, la variation avec $n$ de la probabilité $p(n)$ d'avoir $n$ photons est celle d'une courbe centrée en $\langle n\rangle$ et de largeur $\sqrt{\langle n\rangle}$ (loi de Poisson).

La vitesse de variation de $\left\langle{ }^{n n} \mathbf{S}\right\rangle$ due au processus d'excitation est alors donnée par

$$
\frac{\mathrm{d}^{(1)}}{\mathrm{d} t}<{ }^{n n} \mathrm{~S}>=\frac{1}{T_{\mathrm{p}}} \operatorname{Tr}\left[\left(\sigma_{0} \otimes \sigma_{\mathrm{RF}}\right) P_{n} \mathbf{S} P_{n}\right] .
$$

Le calcul détaillé de (D.4) est effectué en appendice. Nous donnons ici uniquement les résultats de ce calcul, en les exprimant d'ailleurs pour $\frac{\mathrm{d}^{(1)}}{\mathrm{d} t}<{ }^{n n} \mathrm{~S}>$ (grâce aux formules $(\mathrm{B}, 7)$ qui relient ${ }^{n n} \boldsymbol{S}$ et ${ }^{n n} \mathbf{S}$ ). On trouve

$$
\begin{aligned}
& \int \frac{\mathrm{d}^{(1)}}{\mathrm{d} t}<{ }^{n n} S_{z}>=\frac{\mathrm{p}(n)}{T_{\mathrm{p}}}{ }^{\mathrm{ex}} S_{z} \\
& \frac{\mathrm{d}^{(1)}}{\mathrm{d} t}<{ }^{n n} S_{\mathrm{y}}>=\frac{\mathrm{p}(n)}{T_{\mathrm{p}}} J_{0}\left(\frac{\omega_{1}}{\omega}\right)^{\mathrm{ex}} S_{y}
\end{aligned}
$$

soit encore de façon plus condensée

$$
\frac{\mathrm{d}^{(1)}}{\mathrm{d} t}<{ }^{n n} S_{i}>=\frac{\mathrm{p}(n)}{T_{\mathrm{p}}} \frac{\bar{g}_{i j}}{g}{ }^{\mathrm{ex}} S_{j}
$$

Par rapport aux formules $(\mathrm{D}, 1)$, on constate deux différences :

- d'une part le facteur $p(n)$, qui disparaîtra plus loin lorsqu'on sommera les contributions au signal de détection de toutes les multiplicités (on a en effet $\left.\sum p(n)=\right) 1$;

- d'autre part le facteur $J_{0}\left(\omega_{1} / \omega\right)$ qui figure dans le terme d'excitation $\left\langle{ }^{n n} S_{x}\right\rangle$. On voit donc que l'efficacité du pompage sur $\left.{ }^{n n} \boldsymbol{S}\right\rangle$ est réduite par un facteur $J_{0}\left(\omega_{1} / \omega\right)$ dans une direction perpendiculaire au champ «habillant » $H_{1}$ alors qu'elle n'est pas affectée le long de $\mathbf{H}_{1}$.

Remarque. - En toute rigueur on néglige au $2^{\mathrm{e}}$ membre de $(D, 5)$ des termes oscillants aux fréquences multiples de $\omega$ et qui proviennent du caractère cohérent du champ RF. Ces termes ont un effet pratiquement négligeable car leur fréquence est très grande devant la fréquence d'évolution de ${ }^{n n} \boldsymbol{S}$ qui est voisine de $\omega_{0} \ll \omega$ (approximation séculaire).

3) Relaxation.- Nous supposons pour simplifier que la relaxation de l'atome libre est isotrope et décrite par une seule constante de temps, $1 / \Gamma$

$$
\frac{\mathrm{d}^{(2)}}{\mathrm{d} t}<\mathrm{S}>=-\Gamma<\mathrm{S}>\text {. }
$$

(Dans l'exemple d'un niveau atomique excité choisi plus haut, $1 / \Gamma$ est la durée de vie radiative du niveau excité.)

Si le temps de corrélation $\tau_{\mathrm{c}}$ associé aux processus de relaxation est suffisamment court devant $1 / \omega_{1}$ et $1 / \omega$, on peut négliger le couplage entre l'atome et la RF durant le temps des "collisions 》 responsables de la relaxation. On montre alors aisément que l'on peut écrire

$$
\frac{\mathrm{d}^{(2)}}{\mathrm{d} t}<{ }^{n n} \mathrm{~S}>=-\Gamma<{ }^{n n} \boldsymbol{S}>.
$$

Remarque. - Lorsque la condition $\omega_{1} \tau_{\mathrm{c}} \ll 1$ n'est pas remplie, l'« habillage» par le champ de RF a le temps de se manifester pendant le temps de "collision " responsable de la relaxation. Les équations de relaxation de l'atome "habillé » se trouvent alors profondément modifiées et ne peuvent plus se mettre sous la forme $(D, 7)$. Nous n'étudierons pas ici ce cas qui fait l'objet d'une autre publication [14].

En ajoutant les équations $(C, 5)(D, 5)$ et $(D, 7)$ on obtient l'équation pilote dont la solution permet de calculer $\left\langle{ }^{n n} \mathcal{S}>\right.$ à tout instant :

$$
\begin{aligned}
\frac{\mathrm{d}}{\mathrm{d} t}<{ }^{n n} S_{i}> & =\frac{p(n)}{T_{\mathrm{p}}} \frac{\bar{g}_{i j}}{g}{ }^{\text {ex }} S_{j}+ \\
& +g \mu_{\mathrm{B}}\left[<{ }^{n n} \mathrm{~S}>\wedge \mathbf{H}_{0}^{\prime}\right]_{i}-\Gamma<{ }^{n n} S_{i}>.
\end{aligned}
$$

4) Signaux de détection. - Les signaux de détection sont en général proportionnels aux valeurs moyennes d'opérateurs purement atomiques. 
Ainsi, si l'on utilise une méthode de détection radioélectrique, le signal est proportionnel à la composante de $\langle\mathbf{S}\rangle$ perpendiculaire au plan de la bobine de détection.

Dans l'exemple du niveau atomique excité choisi plus haut, on observe la lumière de fluorescence dont l'état de polarısation, l'intensité dépendent de l'orientation $\langle\mathbf{S}\rangle$ du niveau atomique (elles dépendent également de l'alignement et de la population de ce niveau). Le processus d'émission d'un photon est quasi instantané et l'on peut parfaitement négliger tout couplage entre l'atome et la RF au cours de ce processus. Les signaux de détection optique sont donc également pour l'atome "habillé " proportionnels à certaines composantes de $\langle\mathbf{S}\rangle$. Si par exemple, le niveau a un moment cinétique électronique $J=1 / 2$ et que l'on observe la lumière circulaire émise dans une direction $\mathbf{u}$, on détecte $\left\langle S_{u}\right\rangle$, c'est-àdire encore, en utilisant (A.8) $\left.\sum_{n n^{\prime}}<{ }^{n n^{\prime}} S_{u}\right\rangle$. Les termes avec $n \neq n^{\prime}$ correspondent aux parties modulées à la fréquence $\left(n-n^{\prime}\right) \omega$ du signal de détection. Si l'on se limite à la partie basse fréquence du signal, on détecte en fait $\sum_{n}<{ }^{n n} S_{u}>$.

La résolution de l'équation pilote montre que par suite du terme source proportionnel à $p(n)$, le terme $<{ }^{n n} S>$ est proportionnel à $p(n)$. La sommation sur $n$ fait apparaître la quantité $\sum_{n} p(n)=1$, qui rend le résultat indépendant de la fonction $p(n)$, donc de la forme explicite de la distribution des photons dans le champ de radiofréquence.

Remarquons bien que le signal détecté dans la direction $\mathbf{u}$ est proportionnel à $\sum_{n}{ }^{n n} \mathbf{S}_{u}$ et non à $\sum^{n n} S_{u}$, les ${ }^{n n} S$ n'étant que des intermédiaires commodes de calcul. Le calcul d'une expérience nécessite donc la résolution de l'équation de Bloch $(D, 8)$ pour les ${ }^{n n} \mathbf{S}$, puis le passage à l'aide de $(\mathrm{B}, 7)$ des ${ }^{n n} \boldsymbol{S}$ aux ${ }^{n n} \mathrm{~S}$. Le tableau suivant donne, pour un champ $\mathbf{H}_{0}$ nul, et pour différentes directions possibles de pompage et de détection, la dépendance du signal détecté en $\omega_{1} / \omega$; ce tableau se déduit très simplement des équations (D8) et $\mathrm{B}(7)$.

\begin{tabular}{|c|c|c|}
\hline $\begin{array}{l}\text { Direction } \\
\text { Direction } \\
\text { détection }\end{array}$ & ${ }_{1 /} \mathrm{O} z$ & $\perp \mathrm{O} z$ \\
\hline$/ / \mathrm{O} z$ & 1 & $J_{0}\left(\frac{\omega_{1}}{\omega}\right)$ \\
\hline$\perp \mathbf{O} z$ & $J_{0}\left(\frac{\omega_{1}}{\omega}\right)$ & $J_{0}^{2}\left(\frac{\omega_{1}}{\omega}\right)$ \\
\hline
\end{tabular}

E. Vérification expérimentale. - 1) GÉNÉRALITÉS. - Les vérifications expérimentales ont été effectuées dans l'état fondamental de ${ }^{199} \mathrm{Hg}(I=1 / 2)$ pompé optiquement sur la raie $2537 \AA\left({ }^{3}\right)$. La vapeur atomique est contenue dans une cellule de quartz, dont les parois sont chauffées à $200^{\circ} \mathrm{C}$ environ afin d'allonger le temps de relaxation (il est alors de l'ordre de $1 \mathrm{~s}$ ). La cellule est placée à l'intérieur d'un blindage magnétique qui élimine les fluctuations du champ magnétique présent dans le laboratoire. Plusieurs paires de bobines en position de Helmholtz permettent de créer des champs dans les trois directions.

Le champ « habillant » de fréquence $\omega / 2 \pi=265 \mathrm{~Hz}$ est créé dans la direction $\mathrm{Oz}$ par deux de ces bobines. On a veillé à lui donner par des compensations adéquates une polarisation parfaitement linéaire. En effet une polarisation légèrement elliptique, provenant par exemple de courants induits dans certaines parties du montage, modifie très sensiblement les phénomènes observés (voir référence [13]). Les atomes sont pompés optiquement sur la composante hyperfine $F=1 / 2$ de l'état excité par un faisceau polarisé circulairement issu d'une lampe à ${ }^{204} \mathrm{Hg}$ et se propageant dans la direction $\mathrm{Oz}$, Ox ou $\mathrm{Oy}$.

2) Etude expérimentale du tenSeur de Landé. - a. - Il s'agit d'étudier la variation de la fréquence de Larmor des atomes en fonction de l'angle entre le champ magnétique statique $\mathbf{H}_{0}$ et le champ "habillant». Nous avons utilisé une méthode de transitoires: les atomes sont pompés optiquement en champ nul, dans la direction $\mathrm{Oz}$ (ou $\mathrm{Oy}$ ) ; on introduit brusquement $\mathbf{H}_{0}$ dans une direction faisant un angle $\theta$ avec $\mathrm{Oz}$; les atomes se mettent à précesser et cette précession se traduit par une modulation à la fréquence de Larmor de la lumière transmise par la cellule de résonance.

Si l'on connaît $H_{0}$ et $\theta$, la mesure de cette fréquence permet de déterminer le facteur de Landé $\bar{g}(\theta)$ correspondant à la valeur $\omega_{1} / \omega$ du couplage avec la RF.

b. - Le schéma du dispositif expérimental est donné par la figure 3. Le pulse de champ $H_{0}$ est produit en introduisant brusquement dans des bobines d'axe $\mathrm{Oz}$ et $\mathrm{Ox}$ des courants $i_{z}$ et $i_{x}$ dont le rapport est déterminé par un pont diviseur. La mesure de $i_{z}$ et $i_{x}$, et l'étalonnage des bobines permet de connaître $H_{0}$ et $\theta$. On répète l'expérience pour diverses valeurs de $\theta$ et $\omega_{1} / \omega$.

Pour $20^{\circ}<\theta<90^{\circ}$, on pompe dans la direction de $\mathrm{Oz}$; le pompage et la détection (on mesure $\left\langle S_{z}\right\rangle$ ) sont alors indépendants de $\omega_{1} / \omega$ (voir tableau du $\S \mathrm{D}, 4))$. Mais pour $\theta$ petit $\left(\theta<20^{\circ}\right), \mathbf{H}_{0}$

(3) Ce cas ne correspond pas à l'exemple d'un niveau atomique excité envisagé au paragraphe $\mathrm{D}$ précédent. Toutefois à la limite des pompages faibles, les équations d'évolution de $\langle\mathbf{S}\rangle$ sont très semblables à celle de l'orientation d'un état excité. Les calculs du paragraphe D concernant l'efficacité du pompage et de la détection demeurent valables. Mais la détection d'une composante de $\langle\mathbf{S}\rangle$ se fait maintenant en mesurant la lumière absorbée sur le faisceau de pompage ou sur un faisceau de détection. 


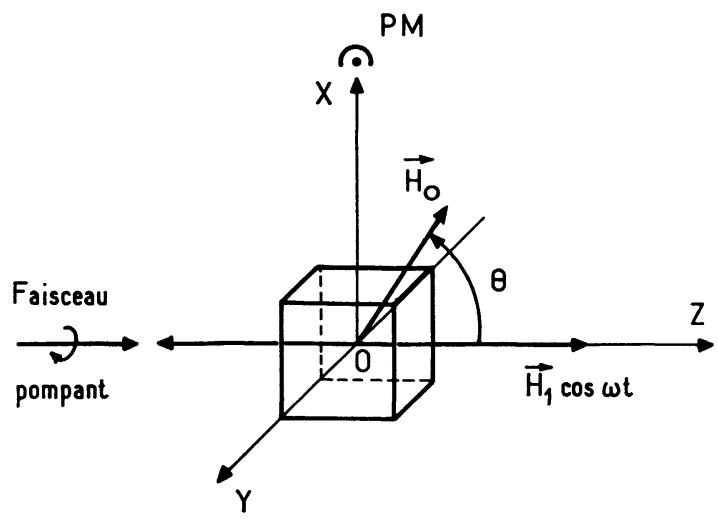

FIG. 3. - Schéma du dispositif expérimental pour l'étude de l'anisotropie du facteur de Landé.

est presque parailèle au pompage, et au cours de la précession $\left\langle S_{z}\right\rangle$ varie très peu. Il devient alors nécessaire de pomper dans une autre direction, Oy par exemple. L'efficacité du pompage décroît alors par un facteur $J_{0}\left(\omega_{1} / \omega\right)$ (voir tableau du $\left.\S \mathrm{D}, 4\right)$. Il en est de même pour la détection puisqu'on détecte $\left\langle S_{y}\right\rangle$ et il devient par suite impossible de faire des mesures pour des valeurs de $\omega_{1} / \omega$ voisines des zéros de $J_{0}$

On suit la précession des atomes en mesurant, avec un photomultiplicateur $\mathrm{PM}$, les variations de la lumière $L_{F}$ diffusée à angle droit par la cellule ; pour ${ }^{199} \mathrm{Hg}$ pompé sur la composante $F=1 / 2, L_{F}$ est strictement proportionnelle à la lumière absorbée par les atomes, $L_{\mathrm{A}}$. Mais on bénéficie ainsi d'un meilleur rapport signal sur bruit.

c. - Les résultats sont présentés sur la figure 4 . L'angle polaire $\theta$ représente l'angle de $\mathbf{H}_{0}$ avec le

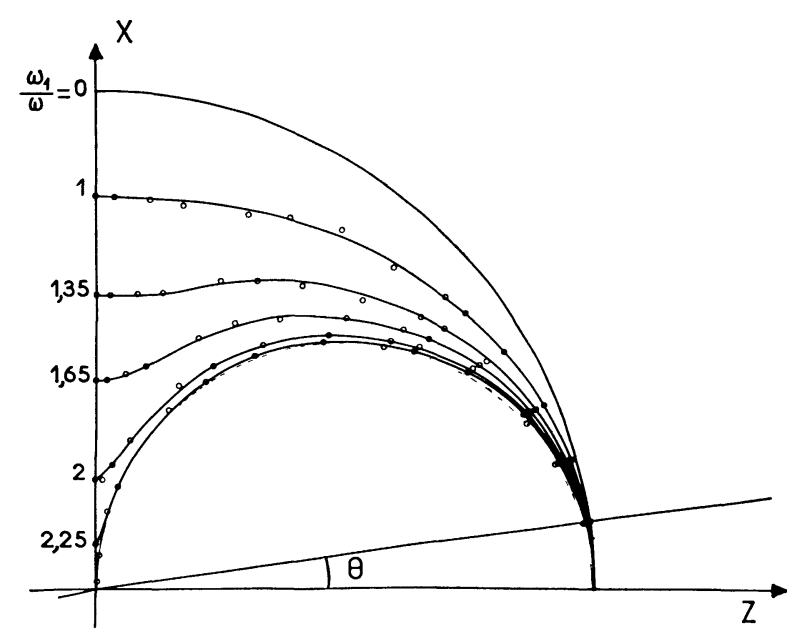

Fig. 4. - Représentation en coordonnées polaires du facteur de Landé de l'atome " habillé " pour diverses valeurs de $\omega_{1} / \omega$.

Les courbes sont théoriques et les points expérimentaux.

champ "habillant»; la longueur du rayon vecteur est proportionnelle au facteur de Landé dans cette direction. Les courbes sont théoriques, tracées pour diverses valeurs de $\omega_{1} / \omega$, à partir de la formule $(\mathrm{B}, 14)$

$$
\bar{g}(\theta)=g \sqrt{\cos ^{2} \theta+J_{0}^{2}\left(\frac{\omega_{1}}{\omega}\right) \sin ^{2} \theta} .
$$

Les points sont expérimentaux. On peut constater que l'accord est excellent.

3) Précession de LARmor de L'Atome « habillé ». - Le but de cette expérience est de mettre en évidence l'ellipticité du mouvement des spins quand ils précessent autour d'un champ perpendiculaire au champ " habillant " (voir paragraphe C).

a. - L'expérience est schématisée sur la figure 5. Les atomes sont orientés le long de $\mathrm{Oz}$ par le faisceau de pompage. On applique brusquement un champ magnétique statique $\mathrm{H}_{0}$ dans la direction $\mathrm{Ox}$ et on suit la précession des spins dans le plan zOy. $\left\langle S_{z}\right\rangle$ est détecté au moyen de la lumière de fluorescence

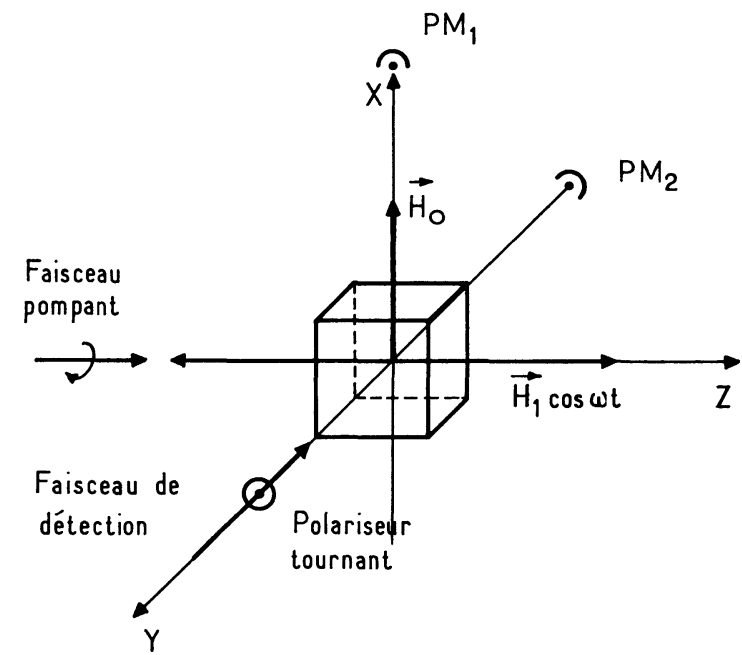

Fig. 5. - Schéma du dispositif expérimental pour l'étude de l'anisotropie de la précession de Larmor $\left(\mathbf{H}_{0}\right.$ perpendiculaire au champ habillant).

$L_{F}$ recueillie par le photomultiplicateur $\mathrm{PM}_{1},\left\langle S_{y}\right\rangle$ au moyen de l'absorption d'un faisceau croisé de détection. En envoyant les deux signaux de détection sur un traceur $\mathrm{XY}$, on reconstitue la précession des spins. Afin de minimiser la perturbation apportée par le faisceau croisé de détection et d'augmenter le rapport signal/bruit, on a modulé sa polarisation entre $\sigma^{+}$et $\sigma^{-}$à une fréquence $\Omega$ grande devant le temps de relaxation et les fréquences de précession des atomes. Le dichroïsme de la vapeur le long de Oy, proportionnel à $\left\langle S_{y}\right\rangle$, se traduit par une modulation à la fréquence $\Omega$ de la lumière du faisceau croisé transmise par la cellule. Une détection en phase de cette modulation donne un signal proportionnel à $\left\langle S_{y}\right\rangle$. Afin que l'enregistreur $X Y$ ne déforme pas les transitoires, nous nous sommes limités à des fréquences de Larmor inférieures à $10 \mathrm{~Hz}$.

b. - Résultats (Fig. 6). - L'expérience proprement dite a été réalisée ainsi : faisant d'abord

$$
\frac{\omega_{1}}{\omega}=0 \text {, }
$$


nous avons réglé l'amplification sur les deux voies de façon à obtenir sur l'enregistrement un mouvement circulaire. En fait, par suite de la relaxation, l'extrémité de $\langle\mathbf{S}\rangle$ décrit non pas un cercle, mais une spirale logarithmique ( $1^{\text {re }}$ courbe, Fig. 6 ; en abscisse

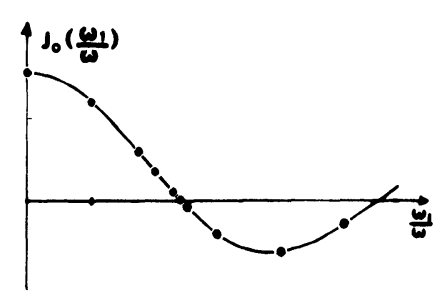

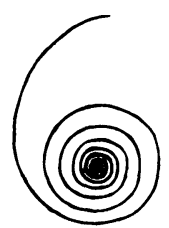

$\frac{\omega_{1}}{\omega}=0$

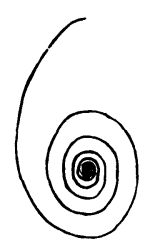

1

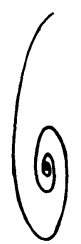

1,75
2
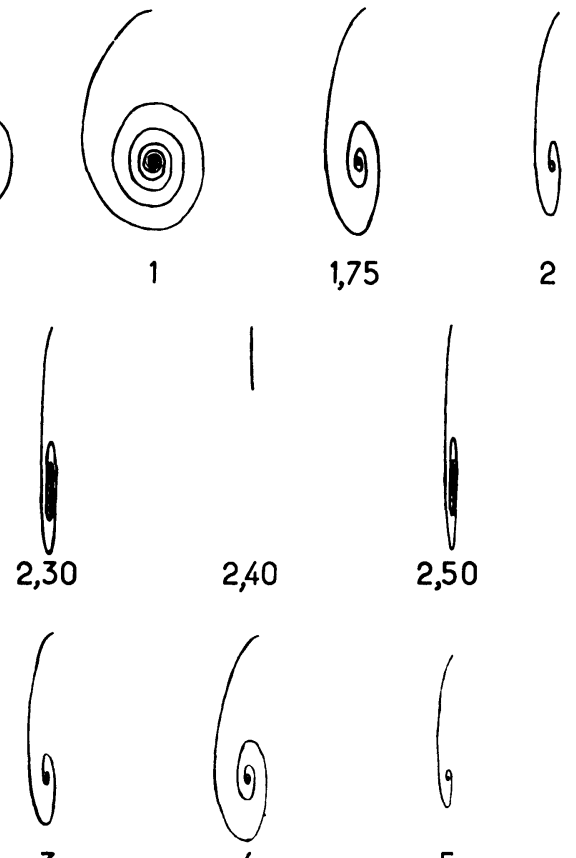

4

2,$4 ; 2,5$ ont été réalisés avec un champ 20 fois plus fort que pour les autres. En effet, sans cette précaution, on ne verrait aucune précession, la fréquence de Larmor $\omega_{0} J_{0}$ devenant très inférieure à la largeur des niveaux).

- Le sens de rotation sur l'ellipse ne change pas lorsqu'on traverse un zéro de la fonction de Bessel. En effet d'après les formules $(B, 7)$ lorsqu'on traverse un zéro de $J_{0}$, le changement de signe de la fréquence de Larmor est accompagné d'un changement de signe de $\left\langle S_{y}\right\rangle$ : il y a compensation des deux effets et le sens de rotation sur l'ellipse demeure le même.

4) RÉSONANCE MAGNÉTIQUE DE L'ATOME « HABILLÉ ». - a. But de l'expérience. - Nous décrivons maintenant une deuxième expérience qui met en évidence le caractère elliptique de la précession de Larmor de l'atome " habillé " dans le champ $\mathbf{H}_{0}$. Un mouvement elliptique est en effet caractérisé par le fait qu'il se décompose en deux mouvements circulaires de même fréquence, mais de sens opposés. Ainsi contrairement à l'atome «nu » qui dans un champ magnétique n'a qu'une seule fréquence propre (la fréquence de Larmor $\omega_{0} / 2 \pi$, définie en grandeur et en signe), un atome " habillé » dans le même champ possède deux fréquences propres de signes opposés $\left(\omega_{0} / 2 \pi\right) J_{0}$ et $-\left(\omega_{0} / 2 \pi\right) J_{0}\left(\omega_{0}\right.$ est multiplié par $J_{0}$ à cause de la modification du facteur de Landé). La mise en évidence de ces deux fréquences propres se fera évidemment en étudiant le spectre de résonance magnétique de l'atome " habillé » obtenu avec un champ de radiofréquence tournant.

Remarque. - Nous introduisons donc ici un deuxième champ de radiofréquence qu'il faut bien distinguer du champ «habillant ». Rappelons que ce dernier est un champ intense, qui modifie les niveaux d'énergie de l'atome. Au contraire le deuxième champ, d'intensité beaucoup plus faible, sert en quelque sorte d'instrument de mesure, destiné à déterminer les fréquences propres du système «atome + champ habillant ». Nous le traiterons toujours classiquement.

b. Principe de l'expérience (Fig. 7). - Les atomes

On constate les phénomènes suivants :

- La précession des spins devient elliptique. L'ellipticité est d'autant plus marquée que $J_{0}\left(\omega_{1} / \omega\right)$ est plus petit : à la limite $J_{0}=0$, l'ellipse devient une droite. Le rapport mesuré des axes de l'ellipse est conforme aux prévisions théoriques comme le montre le tableau suivant :

\begin{tabular}{l|ccccc}
$\omega_{1} / \omega$ & 0,5 & 1 & 1,5 & 1,75 & 2 \\
\cline { 1 - 6 } $\begin{array}{l}b / a \text { théorique } \\
b / a \text { exp. }\end{array}$ & 0,94 & 0,76 & 0,51 & 0,37 & 0,22 \\
0,90 & 0,76 & 0,50 & 0,34 & 0,18
\end{tabular}

- Lorsque $J_{0}$ décroît, le nombre de tours réalisés par $\langle\mathbf{S}\rangle$ pendant le temps de relaxation décroît : ceci signifie que la vitesse de précession diminue. (Les trois enregistrements faits pour $\omega_{1} / \omega=2,3$;

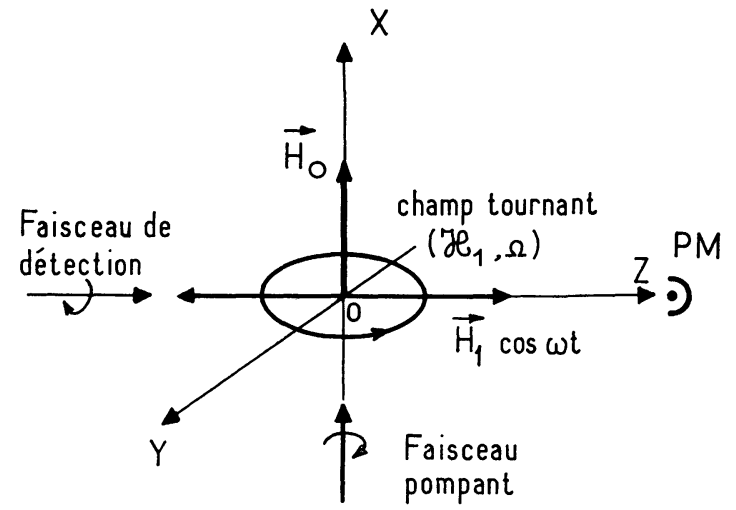

Fig. 7. - Schéma du dispositif expérimental pour l'étude de la résonance magnétique de l'atome « habillé ». 
« habillés » par le champ $\mathbf{H}_{1} \cos \omega \mathrm{t}$, sont placés dans un champ magnétique $\mathbf{H}_{0}$ parallèle à $\mathbf{O x}$. Ils sont orientés le long de $\mathbf{H}_{0}$ par un faisceau de pompage optique et soumis à l'action d'un champ de RF circulaire droit d'intensité $\mathcal{H}_{1}$ faible, tournant dans le plan $\mathrm{yOz}$ à la vitesse angulaire $\Omega$. La fréquence $\Omega / 2 \pi=20 \mathrm{~Hz}$ est petite devant $\omega / 2 \pi=265 \mathrm{~Hz}$. Le champ circulaire est réalisé en envoyant dans deux bobines perpendiculaires des courants alternatifs déphasés de $\pi / 2$. Un faisceau croisé de détection, parallèle à $\mathrm{Oz}$, permet d'observer l'aimantation transversale qui apparaît lorsqu'on passe à résonance.

- Pour un atome «nu» $\left(\omega_{1}=0\right)$ nous savons qu'il y a résonance lorsque le champ de RF tourne à la même vitesse et dans le même sens que le moment magnétique de l'atome, c'est-à-dire lorsque $\omega_{0}=\Omega$. Aucune résonance n'apparaît en $\omega_{0}=-\Omega$.

- Pour un spin " habillé », il y a résonance lorsque $\Omega$ est égale à l'une ou l'autre des deux fréquences propres, donc pour $\omega_{0} J_{0}=\Omega$ et $\omega_{0} J_{0}=-\Omega$. L'apparition dans un champ purement circulaire droit d'une résonance «extraordinaire» pour

$$
\omega_{0} J_{0}=-\Omega \text {, }
$$

est donc directement liée au caractère elliptique de la précession de Larmor de l'atome « habillé ».

Remarque. - On sait que pour l'atome «nu», la résonance $\omega_{0}=-\Omega$ est interdite, parce qu'elle violerait le principe de conservation du moment cinétique. Pour l'atome « habillé », le champ " habillant» constitue un "réservoir » de moment cinétique, et c'est ce qui explique la non-conservation apparente du moment cinétique pour la résonance $\omega_{0} J_{0}=-\Omega$. En fait, il y a conservation du moment cinétique du système total : " atome + champ $\mathscr{H}_{1}+$ champ $H_{1}$ ».

c. Problème équivalent. - Le calcul exact des signaux observés se fait évidemment en utilisant les concepts de champ et de moment cinétique fictifs introduits au $\S \mathrm{B}$. Le problème équivalent est celui de l'étude du spectre de résonance d'un atome libre dans un champ $H_{0}^{\prime}=J_{0} H_{0}$, obtenu avec un champ de $\mathrm{RF}$ elliptique $\mathcal{H}_{1}^{\prime}$ de grand axe $\mathcal{H}_{1}$ parallèle à $\mathrm{Oz}$ et de petit axe $\mathcal{H}_{1} J_{0}$ parallèle à $O y$. Ce champ de RF elliptique se décompose en deux champs de RF circulaires droit et gauche d'amplitudes respectives $\mathscr{H}_{1}\left(1+J_{0}\right) / 2$ et $\mathcal{H}_{1}\left(1-J_{0}\right) / 2$. Ces deux composantes induisent respectivement les résonances dans les champs $+\Omega / J_{0}$ et $-\Omega / J_{0}$.

d. Résultats expérimentaux. - Les résultats expérimentaux sont présentés sur la figure 8. En l'absence d' « habillage » $\left(\omega_{1} / \omega=0\right)$, seule la résonance $\omega_{0}=\Omega$ apparaît. C'est d'ailleurs en annulant la résonance $\omega_{0}=-\Omega$ que nous avons réglé la polarisation circulaire droite du champ $\mathscr{H}_{1}$. Lorsqu'on introduit le champ « habillant» $\left(\omega_{1} / \omega \neq 0\right)$, on constate les faits suivants :
- la résonance ordinaire est déplacée vers les champs forts : elle est maintenant centrée en

$$
\omega_{0}=\frac{\Omega}{J_{0}}
$$

- il apparaît, comme prévu, une résonance dans le champ $\omega_{0}=-\Omega / J_{0}$.

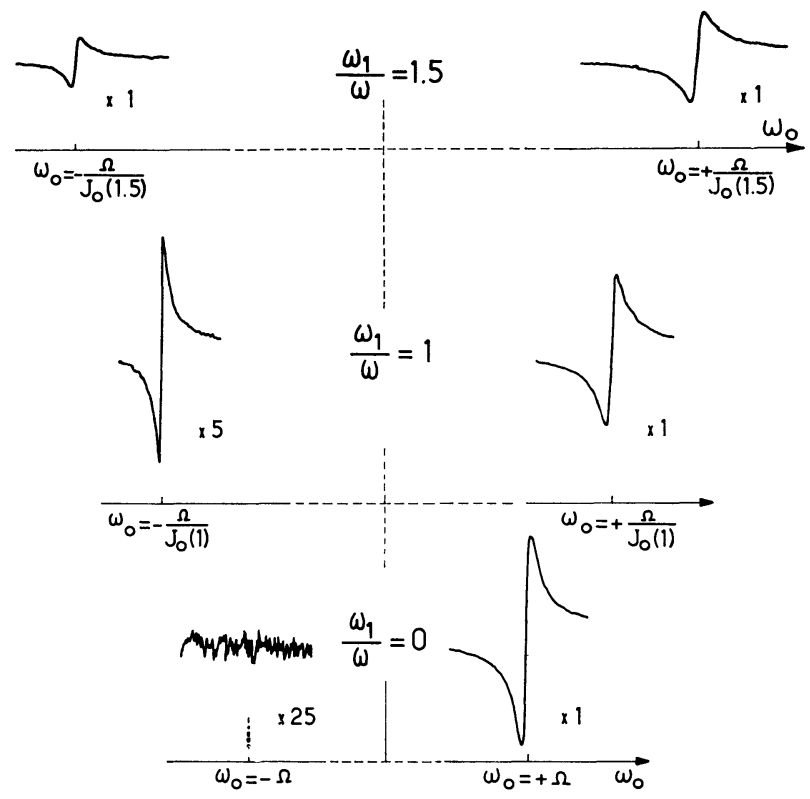

FIG. 8. - Spectre de résonance magnétique de l'atome « habillé » pour diverses valeurs de $\omega_{1} / \omega$. La sensibilité de la détection est indiquée pour chacune des résonances.

L'évolution de ces deux résonances lorsqu'on fait varier $\omega_{1} / \omega$ est conforme aux prévisions de la théorie esquissée au $\S \mathrm{E}, 4 c$. La résonance ordinaire est induite par la composante circulaire droite $\mathscr{H}_{1}\left(1+J_{0}\right) / 2$ du champ de RF fictif. $\mathcal{H}_{1}$ est fixe, juste assez intense pour saturer la résonance de l'atome « nu ». Lorsqu'on augmente $\omega_{1} / \omega$, on constate une diminution de l'intensité de la résonance ordinaire liée à la décroissance de $\left(1+J_{0}\right) / 2$. Au contraire, l'intensité de la résonance extraordinaire, induite par la composante circulaire gauche $\mathscr{H}_{1}\left(1-J_{0}\right) / 2$, croît rapidement avec $\omega_{1} / \omega$, jusqu'à atteindre une intensité voisine de celle de la résonance ordinaire.

En conclusion, nous avons montré que la direction privilégiée introduite par le champ de RF entraîne une anisotropie des propriétés magnétiques de l'atome « habillé »: Tenseur de Landé, précession de Larmor elliptique.

Pour interpréter quantitativement ces effets, nous avons introduit un formalisme opératoriel qui apporte une certaine systématique dans le calcul des expériences de pompage optique sur des atomes « habillés » :

- Il permet d'étudier séparément l'influence de l'« habillage » sur chacune des trois étapes du cycle de pompage : excitation, évolution propre, détection.

- Il permet de ramener le problème de l'évolution de l'atome " habillé » dans un champ $\mathbf{H}_{0}$ à celui d'un 
atome « nu » dans un champ fictif, $\mathbf{H}_{0}^{\prime}$, se déduisant simplement de $\mathbf{H}_{0}$.

L'utilisation de ce formalisme dans des cas plus complexes (collisions d'échange entre atomes alcalins «habillés » [8], application simultanée de plusieurs champs de RF à un même atome [15]) a revélé qu'il était d'une grande souplesse.

APPENDICE. - Calcul du terme décrivant la préparation de $\left\langle{ }^{n n} S\right\rangle$. Généralisation à un opérateur tensoriel irréductible. - 1) RAPPEL DES HYPOTHÈSES. - Le terme de préparation correspond à une matrice densité $\sigma_{0}$ pour l'atome «nu ». On pose

$$
{ }^{\mathrm{ex}} \mathbf{S}=\operatorname{Tr}\left(\sigma_{0} \mathbf{S}\right) \text {. }
$$

- Pour l'atome «habillé », la matrice densité correspondante est $\sigma_{0} \otimes \sigma_{\mathrm{RF}}$, où $\sigma_{\mathrm{RF}}$ est la matrice densité du champ de radiofréquence à l'instant $t$.

- On suppose le champ de RF décrit par un état cohérent de Glauber

$$
\left.\left|\alpha(t)>=\sum_{r} c_{r}(\alpha) \mathrm{e}^{-i r \omega t}\right| r\right\rangle .
$$

$\mid r>$ est l'état du champ avec $r$ photons présents,

$$
c_{r}(\alpha)=\mathrm{e}^{-\alpha^{2} / 2} \frac{\alpha^{r}}{\sqrt{r !}}
$$

(on prend $\alpha$ réel $>0$ ). La probabilité $p(r)=\left|c_{r}(\alpha)\right|^{2}$ de trouver $\mathrm{r}$ photons de RF est une loi de Poisson centrée en $\langle n\rangle=\left.K\right|^{2}$, nombre moyen de photons, et de largeur $\sqrt{\langle n\rangle}$. Pour les intensités de $\mathrm{RF}$ utilisées, $\langle n\rangle$ est très grand $\left(\approx 10^{23} / \mathrm{cm}^{3}\right)$. est

- Le terme de préparation de l'observable ${ }^{n n^{\prime}} \mathbf{S}$

$$
\frac{\mathrm{d}^{(1)}}{\mathrm{d} t}<{ }^{n n^{\prime}} \mathrm{S}>=\frac{1}{T_{\mathrm{p}}} \operatorname{Tr}\left(\sigma_{0} \otimes \sigma_{\mathrm{RF}} P_{n} \mathbf{S} P_{n^{\prime}}\right)
$$

Nous nous proposons de calculer cette quantité en fonction de ex $S$ et des paramètres du champ de RF.

2) Introduction DE $S_{\mathrm{q}}$. TAuX D'excitation DE $<{ }^{n}{ }^{\prime} S_{\mathrm{q}}>$. - Il est commode d'introduire les composantes $S_{\mathrm{q}}(q=1,0,-1)$ définies par $S_{ \pm 1}=S_{\mathrm{x}} \pm i S_{\mathrm{y}}$, $S_{0}=S_{\mathrm{z}} \cdot{ }^{\text {ex }} S_{\mathrm{q}}$ et ${ }^{n n^{\prime}} S_{\mathrm{q}}$ se définissent par des relations analogues. La propriété essentielle de $S_{\mathrm{q}}$ est de relier $\left|m^{\prime}\right\rangle$ uniquement à $\left|m^{\prime}+q\right\rangle$

$$
\begin{aligned}
<m\left|S_{q}\right| m^{\prime}> & =<m^{\prime}+q\left|S_{q}\right| m^{\prime}>\delta_{m, m^{\prime}+q} \\
{ }^{e x} S_{q}= & \operatorname{Tr}\left(\sigma_{0} S_{q}\right)= \\
= & \sum_{m^{\prime}}<m^{\prime}\left|\sigma_{0}\right| m^{\prime}+q><m^{\prime}+q\left|S_{q}\right| m^{\prime}> \\
\frac{\mathrm{d}^{(1)}}{\mathrm{d} t}<{ }^{n n^{\prime}} S_{q}> & =\frac{1}{T_{\mathrm{p}}} \operatorname{Tr}\left(\sigma_{0} \otimes \sigma_{\mathrm{RF}} P_{n} S_{q} P_{n^{\prime}}\right) \\
= & \frac{1}{T_{\mathrm{p}}} \sum_{m m^{\prime}}<m^{\prime}, \bar{n}_{m^{\prime}}^{\prime}\left|\sigma_{0} \otimes \sigma_{\mathrm{RF}}\right| m, \bar{n}_{m}> \\
& \times<m, \bar{n}_{m}\left|S_{q}\right| m^{\prime}, \bar{n}_{m^{\prime}}^{\prime}>. \quad \text { (Ap.2) }
\end{aligned}
$$

On peut séparer les éléments de matrices atomiques et de radiofréquence

$$
\begin{aligned}
& \quad \frac{\mathrm{d}^{(1)}}{\mathrm{d} t}<{ }^{n n^{\prime}} S_{q}> \\
& =\frac{1}{T_{\mathrm{p}}} \sum_{m m^{\prime}}<m^{\prime}\left|\sigma_{0}\right| m><\bar{n}_{m^{\prime}}^{\prime}\left|\sigma_{\mathrm{RF}}\right| \bar{n}_{m}> \\
& \quad \times<m\left|S_{q}\right| m^{\prime}><\bar{n}_{m} \mid \bar{n}_{m^{\prime}}^{\prime}> \\
& =\frac{1}{T_{\mathrm{p}}} \sum_{m^{\prime}}<m^{\prime}\left|\sigma_{0}\right| m^{\prime}+q><m^{\prime}+q\left|S_{q}\right| m^{\prime}> \\
& \quad \times<\bar{n}_{m^{\prime}}^{\prime}\left|\sigma_{\mathrm{RF}}\right| \bar{n}_{m^{\prime}+q}><\bar{n}_{m^{\prime}+q} \mid \bar{n}_{m^{\prime}}^{\prime \mathbf{T}}>\text { (Ap.3) }
\end{aligned}
$$

en utilisant la propriété de $S_{q}$.

$$
<\bar{n}_{m^{\prime}+q} \mid \bar{n}_{m^{\prime}}^{\prime}>=J_{n-n^{\prime}}\left(q \frac{\omega_{1}}{\omega}\right)
$$

est indépendant de $m^{\prime}$; nous allons voir au paragraphe suivant qu'il en est de même $\left\langle\bar{n}_{m^{\prime}}^{\prime}\left|\sigma_{\mathrm{RF}}\right| \bar{n}_{m^{\prime}+q}\right\rangle$. On en déduit que

$$
\begin{aligned}
\frac{\mathrm{d}^{(1)}}{\mathrm{d} t}<{ }^{n n^{\prime}} S_{q}>= & \frac{{ }^{\mathrm{ex}} S_{q}}{T_{\mathrm{p}}} J_{n-n^{\prime}}\left(q \frac{\omega_{1}}{\omega}\right) \times \\
& \times<\bar{n}_{m^{\prime}}^{\prime}\left|\sigma_{\mathrm{RF}}\right| \bar{n}_{m^{\prime}+q}>.
\end{aligned}
$$

3) CALCUL de $<\bar{n}_{m^{\prime}}^{\prime}\left|\sigma_{\mathrm{RF}}\right| \bar{n}_{m}>$. - En utilisant l'expression de $\mid \alpha>$ et un calcul analogue à celui de l'appendice de la référence [9], on démontre que

$$
<\bar{n}_{m}|\alpha\rangle=\sum_{r} c_{r}(\alpha) \mathrm{e}^{-i r \omega t} J_{n-r}\left(m \frac{\omega_{1}}{\omega}\right) \text {. }
$$

D'où l'on déduit

$$
\begin{array}{r}
\left.<\bar{n}_{m^{\prime}}^{\prime}\left|\sigma_{\mathrm{RF}}\right| \bar{n}_{m}\right\rangle=\sum_{r, r^{\prime}} c_{r}(\alpha) \mathrm{e}^{i r \omega t} J_{n-r} \times \\
\times c_{r^{\prime}}(\alpha) \mathrm{e}^{-i r^{\prime} \omega t} J_{n^{\prime}-r^{\prime}}\left(m^{\prime} \frac{\omega_{1}}{\omega}\right) .
\end{array}
$$

(Rappelons que $c_{r}(\alpha)$ est réel.)

On voit facilement sur le développement en série des fonctions de Bessel (voir par exemple A. Angot «Compléments de mathématiques » Paris, p. 365) que

$$
J_{n-r}\left(m \frac{\omega_{1}}{\omega}\right)
$$

tend rapidement vers 0 lorsque $n-r$ est très supérieur à $m\left(\omega_{1} / \omega\right)$. Ordinairement $m\left(\omega_{1} / \omega\right)$ ne dépasse pas 10 ou 20 , de sorte que seuls sont non nuls, les termes où $r$ et $r^{\prime}$ diffèrent respectivement de $n$ et $n^{\prime}$ par des quantités au plus de l'ordre de 100 à 1000 . Or $c_{r}(\alpha)$ varie de façon significative seulement si $r$ varie d'une quantité de l'ordre de $\sqrt{\langle n\rangle}$ qui est considérablement plus grande. On pourra donc remplacer $c_{r}(\alpha)$ et $c_{r^{\prime}}(\alpha)$ par $c_{n}(\alpha)$ et $c_{n^{\prime}}(\alpha)$ dans l'expression (Ap. 6). On peut alors récrire : 


$$
\begin{gathered}
<\bar{n}_{m^{\prime}}^{\prime}\left|\sigma_{\mathrm{RF}}\right| \bar{n}_{m}>=c_{n}(\alpha) c_{n^{\prime}}(\alpha) \exp \left[i\left(n-n^{\prime}\right) \omega t\right] \\
\times\left(\sum_{r} \exp [-i(n-r) \omega t] J_{n-r}\left(m \frac{\omega_{1}}{\omega}\right)\right) \\
\times\left(\sum_{r^{\prime}} \exp \left[+i\left(n^{\prime}-r^{\prime}\right) \omega t\right] J_{n^{\prime}-r^{\prime}}\left(m^{\prime} \frac{\omega_{1}}{\omega}\right)\right) \\
=c_{n}(\alpha) c_{n^{\prime}}(\alpha) \exp \left[i\left(n-n^{\prime}\right) \omega t\right] \\
\quad \times \exp \left(i\left(m^{\prime}-m\right) \frac{\omega_{1}}{\omega} \sin \omega t\right) .
\end{gathered}
$$

On a en effet

$$
\exp [i x \sin \theta]=\sum_{r=-\infty}^{+\infty} J_{r}(x) \exp [i r \theta] .
$$

Pour les valeurs de $n-n^{\prime}$ qui nous intéresseront, on aura toujours $\left|n-n^{\prime}\right| \ll \sqrt{\langle n\rangle}$ de sorte que $c_{n}(\alpha) \approx c_{n^{\prime}}(\alpha)$. On a alors $c_{n}(\alpha) c_{n^{\prime}}(\alpha) \approx p(n)$

$$
\begin{aligned}
<\bar{n}_{m^{\prime}}^{\prime}\left|\sigma_{\mathrm{RF}}\right| \bar{n}_{m} & >=p(n) \exp \left[i\left(n-n^{\prime}\right) \omega t\right] \times \\
& \times \exp \left(i\left(m^{\prime}-m\right) \frac{\omega_{1}}{\omega} \sin \omega t\right) .
\end{aligned}
$$

4) RÉSUltat FINAL. - En reportant le résultat précédent dans (Ap. 4) on trouve

$$
\begin{aligned}
& \frac{\mathrm{d}^{(1)}}{\mathrm{d} t}<{ }^{n n^{\prime}} S_{q}>=\frac{{ }^{\mathrm{ex}} S_{q}}{T_{\mathrm{p}}} p(n) J_{n-n^{\prime}}\left(q \frac{\omega_{1}}{\omega}\right) \times \\
& \quad \times \exp \left[i\left(n-n^{\prime}\right) \omega t\right] \exp \left(-i q \frac{\omega_{1}}{\omega} \sin \omega t\right) .
\end{aligned}
$$

Si l'on fait l'approximation séculaire, on ne garde de ce terme que la partie modulée à $\left(n-n^{\prime}\right) \omega$ c'est-àdire la fréquence d'évolution de $\left\langle{ }^{n^{\prime}} S_{q}\right\rangle$. Il reste donc

$$
\begin{aligned}
& \frac{\mathrm{d}^{(1)}}{\mathrm{d} t}<{ }^{n n^{\prime}} S_{q}>=\frac{{ }^{\mathrm{ex}} S_{q}}{T_{p}} p(n) J_{n-n^{\prime}}\left(q \frac{\omega_{1}}{\omega}\right) \times \\
& \times \exp \left[i\left(n-n^{\prime}\right) \omega t\right] J_{0}\left(q \frac{\omega_{1}}{\omega}\right) .
\end{aligned}
$$

Dans le cas particulier d'une observable «basse fréquence $»\left(n=n^{\prime}\right)$

$$
\frac{\mathrm{d}^{(1)}}{\mathrm{d} t}<{ }^{n n} S_{q}>=\frac{{ }^{\mathrm{ex}} S_{q}}{T_{\mathrm{p}}} p(n) J_{0}^{2}\left(q \frac{\omega_{1}}{\omega}\right)
$$

d'où l'on tire

$$
\frac{\mathrm{d}^{(1)}}{\mathrm{d} t}<{ }^{n n} \mathrm{~S}_{q}>=\frac{{ }^{\mathrm{ex}} S_{q}}{T_{\mathrm{p}}} p(n) J_{0}\left(q \frac{\omega_{1}}{\omega}\right) .
$$

Ces résultats se généralisent [voir référence 13] à un opérateur tensoriel irréductible quelconque $T_{q}^{(k)}$. Si

$$
\begin{gathered}
\operatorname{Tr}\left(\sigma_{0} T_{q}^{(k)}\right)={ }^{\mathrm{ex}} T_{q}^{(k)} \\
\frac{\mathrm{d}^{(1)}}{\mathrm{d} t}<{ }^{n n^{\prime}} T_{q}^{(k)}>=\frac{{ }^{\mathrm{ex}} T_{q}^{(k)}}{T_{\mathrm{p}}} p(n) J_{n-n^{\prime}}\left(q \frac{\omega_{1}}{\omega}\right) \times \\
\times \exp \left[i\left(n-n^{\prime}\right) \omega t\right] \exp \left(-i q \frac{\omega_{1}}{\omega} \sin \omega t\right) .
\end{gathered}
$$

Soit en passant à l'opérateur fictif ${ }^{n n^{\prime}} \mathfrak{G}_{q}^{(k)}$ défini par

$$
{ }^{n n^{\prime}} T_{q}^{(k)}=J_{n-n^{\prime}}\left(q \frac{\omega_{1}}{\omega}\right)^{n n^{\prime}} \mathcal{C}_{q}^{(k)}
$$

et en faisant l'approximation séculaire :

$$
\begin{aligned}
\frac{\mathrm{d}^{(1)}}{\mathrm{d} t}<{ }^{n n^{\prime}} T_{q}^{(k)}>=\frac{{ }^{\mathrm{ex}} T_{q}^{(k)}}{T_{\mathrm{p}}} & p(n) J_{0}\left(q \frac{\omega_{1}}{\omega}\right) \times \\
& \times \exp \left[i\left(n-n^{\prime}\right) \omega t\right] .
\end{aligned}
$$

\section{Bibliographie}

[1] Cohen-Tannoudi (C.), Haroche (S.), C. R. Acad. Sci. Paris, 1966, 262, 37.

[2] Cohen-Tannoudi (C.), Haroche (S.), J. Physique, $1969,30,125$ et 153.

[3] Cohen-Tannoudj (C.), Haroche (S.), dans « Polarisation, Matière et Rayonnement ), livre de jubilé en l'honneur d'Alfred Kastler, édité par la Société Française de Physique, P. U. F., 1969.

[4] Cohen-Tannoudji (C.), Cargèse Lectures in Physics, édité par M. Jean (Gordon and Breach), New-York, 1968, 2, 347.

[5] Autler (S. H.) et Townes (C. H.), Phys. Rev., 1955, 100, 203.

[6] Cohen-Tannoudji (C.), Haroche (S.), C. R. Acad. Sci. Paris, 1966, 262, 268. Voir aussi références [2], [3], [4].
[7] Haroche (S.), Cohen-Tannoudi (C.), Audoin (C.) et Schermann (J. P.), Phys. Rev. Lett., 1970, 24, 816.

[8] Haroche (S.), Cohen-Tannoudj (C.), Phys. Rev. Lett., 1970, 24, 974.

[9] Polonsky (N.), Cohen-Tannoudji (C.), J. Physique, 1965, 26, 409.

[10] Glauber (R. J.), Phys. Rev., 1963, 131, 6, 2766-2788.

[11] Pour la définition de la projection d'un opérateur dans un niveau hyperfin $F$ ou entre deux niveaux hyperfins $F$ et $F^{\prime}$ voir Омоnт (А.), J. Physique, 1965, 26, 26.

[12] Cohen-Tannoudji (C.), Thèse, Paris, 1962; Ann. Phys., 1962, 7, 433 et 469.

[13] Landré (C.), Thèse de $3^{\mathrm{e}}$ Cycle, Paris, 1970, non publiée.

[14] HaRoche (S.), Cohen-Tannoudji (C.), à paraître.

[15] Dupont-Roc (J.), CoHEn-TANNOUdJi (C.), à paraître. 